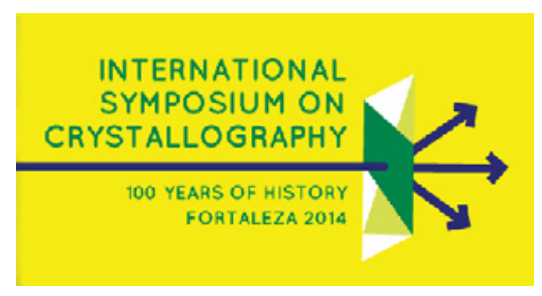

\title{
EQUAL OPPORTUNITY CRYSTALLOGRAPHY OF OXIDE SURFACES
}

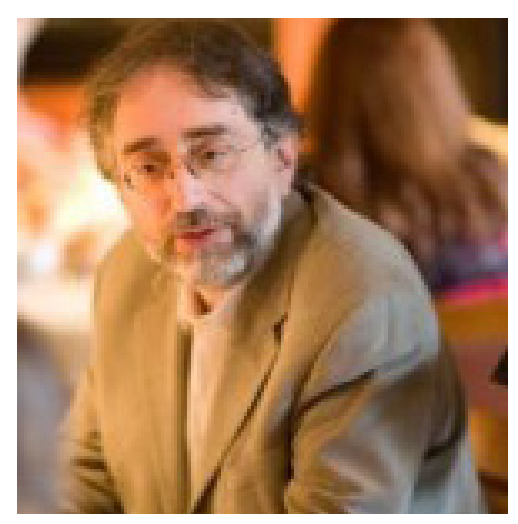

\author{
Laurence D. Marks
}

Department of Materials Science and Engineering, Northwestern University, Evanston, IL 60201

Oxide surfaces represent a number of unique challenges, needing a more flexible approach than for bulk materials. While $x$-ray methods in reciprocal space can be the most accurate and easy to model, they suffer from weak scattering from a thin surface so the errors can be large. Electron based methods in reciprocal space have much larger signals, but also can have dynamical effects which are harder (but not impossible) to model. Crystallography in real-space, for instance scanning probe and/or electron microscopies can be invaluable, but with scanning probe methods there are ambiguities in interpretation and electron-based methods for surfaces tend to have weak signals. Crystallography in-silico is useful, but one has to recognize that methods such as DFT for oxides are full of traps for the unwary and can be quite inaccurate unless more than normal care is taken.

The most effective approach is to take an equal opportunity approach, combining different techniques; follow the science not the tool. By this strategy we have been fairly successful over the last decade in understanding a fair amount about the surface of SrTiO3. Despite being a simple perovskite in the bulk, at the surface it is probably the most complicated material yet known with about ten different structures on each of the main faces, (001), (110) and (111). From a range of techniques it is now starting to emerge that there is a common theme to these surfaces involving local ordering of octahedral or tetrahedral units, not too different from what is found in many bulk oxides. 